\title{
Influencia de las condiciones de procesamiento sobre las propiedades dieléctricas y microestructurales de cerámicos de $\mathrm{K}_{1 / 2} \mathrm{Na}_{1 / 2} \mathrm{NbO}_{3}$
}

\author{
L. RAMAJO, M.M. REBOREDO, M.S. CASTRO
}

Instituto de Investigaciones en Ciencia y Tecnología de Materiales (INTEMA) (CONICET - Universidad Nacional de Mar del Plata). Argentina.

\begin{abstract}
El objetivo de este trabajo es analizar diferentes condiciones de procesamiento del $\mathrm{K}_{1 / 2} \mathrm{Na}_{1 / 2} \mathrm{NbO}_{3}(\mathrm{KNN})$ que permitan obtener un material con buenas propiedades y libre de fases secundarias. Se implementó el método de activación mecanoquímica de los precursores y posterior reacción en estado sólido. Se analizaron las principales condiciones de procesamiento (tiempo de molienda, temperaturas de calcinación y sinterizado), y se determinaron las propiedades dieléctricas y microestructurales de cada sistema. Se encontró que las temperaturas máximas de calcinación estuvieron limitadas por la descomposición de los constituyentes. También se observó que las pastillas sinterizadas a $1125^{\circ} \mathrm{C}$ registraron mayor densidad, mayor permitividad y una microestructura homogénea. En general, se observó que los sistemas calcinados a mayores temperaturas y por tiempos mayores tuvieron mejores propiedades.
\end{abstract}

Palabras claves: Piezoeléctricos, procesamiento, propiedades dieléctricas, $\mathrm{K}_{1 / 2} \mathrm{Na}_{1 / 2} \mathrm{NbO}_{3}$.

Effects of processing conditions on microstructural and dielectrical properties of $\mathrm{K}_{1 / 2} \mathrm{Na}_{1 / 2} \mathrm{NbO}_{3}$ ceramics

The objective of this work was to analyze different processing condition of $\mathrm{Na}_{1 / 2} \mathrm{~K}_{1 / 2} \mathrm{NbO}_{3}(\mathrm{KNN})$ in order to obtain a material with good properties and without secondary phases. Solid state reaction method was used and the influence of milling time, calcination temperature and time, and sintering temperature was analyzed. It was found that sintering temperatures were limited by the component decomposition. Also, it was observed that samples sintered at $1125^{\circ} \mathrm{C}$ showed the highest density and permittivity with a homogeneous structure. In general, samples calcined at higher temperatures and times showed the best properties.

Keywords: Piezoelectric, processing, dielectric properties, $\mathrm{K}_{1 / 2} \mathrm{Na}_{1 / 2} \mathrm{NbO}_{3}$

\section{INTRODUCCIÓN}

Los cerámicos de zirconato-titanato de plomo $(\mathrm{Pb}(\mathrm{Zr}, \mathrm{Ti})$ $\mathrm{O}_{3}$-PZT) son los materiales piezoeléctricos más utilizados (60\% del mercado) y estudiados para aplicaciones tales como actuadores, sensores, transductores o vibradores (1-4). En general, se caracterizan por tener alta sensibilidad, confiabilidad y la capacidad de operar en un amplio intervalo de temperaturas sin modificar sus características piezoeléctricas.

Los PZT se fabrican generalmente a partir de la técnica de mezcla de óxidos, la que es relativamente simple y económica. Sin embargo, este material posee contenidos de óxido de plomo $(\mathrm{PbO})$ de aproximadamente 60 a $70 \%$. El plomo y sus compuestos son considerados tóxicos y peligrosos, no sólo por la polución directa que genera el proceso de manufactura y maquinado de los compuestos, sino también porque los productos conteniendo PZT no pueden ser reciclados. Otro inconveniente surge de la alta dependencia del comportamiento piezoeléctrico del material con la composición del PZT que puede ser afectada por la volatilización del $\mathrm{PbO}$ (5).
Por lo expuesto, desde hace algunos años se ha comenzado a analizar diferentes alternativas para reducir el uso de plomo a partir del desarrollo de una nueva familia de materiales piezoeléctricos que reemplacen a los tradicionales PZT. Así es que, se están estudiando materiales cerámicos basados en bismuto con estructuras en capas (6), en titanatos con estructura tipo perovskita $\left(\mathrm{Bi}_{1 / 2} \mathrm{~A}_{1 / 2}\right) \mathrm{TiO}_{3}(\mathrm{~A}=\mathrm{Na}, \mathrm{K})(7)$, y también en sistemas conformados por niobiatos alcalinos $\left[\left(\mathrm{K}_{1-x^{\prime}} \mathrm{Na}_{\mathrm{x}}\right) \mathrm{NbO}_{3}\right](\mathrm{KNN})$ (8-10). Estos últimos, han demostrado tener propiedades piezoeléctricas equivalentes a las de los PZT (11), lo que permite su utilización en la fabricación de transductores y otro tipo de dispositivos electrónicos. Al igual que el PZT los cerámicos KNN presentan una transición de fase morfológica (dos fases ortorrómbicas a $50 \% \mathrm{~K}$ y $50 \%$ $\mathrm{Na}$ ), que incrementa las propiedades de las composiciones cercanas a esta transición. Dicha transformación de fase puede ser fácilmente modificada reemplazando algunos de los cationes A y B de la estructura tipo perovskita $\left(\mathrm{ABO}_{3}\right)$, incrementando la temperatura de Curie y aumentando la constante piezoeléctrica (12). 
La principal desventaja del KNN es el cuidado que se debe tener con los materiales de partida, la sensibilidad de las propiedades con la composición y el complejo proceso de densificación (13-14). Por ello, se encuentran en proceso de análisis varias técnicas tales como prensado en caliente (15) y sinterizado por descarga de plasma (16), con el objetivo de producir cerámicos KNN de alta densidad, con buen control microestructural y buenas propiedades. No obstante, estas técnicas son poco útiles a escala industrial debido a sus altos costos o baja productividad, por lo que es necesario desarrollar nuevos procesos que permitan obtener el material a bajas temperaturas y con altos niveles de densificación. En este sentido, se ha determinado que la activación mecanoquímica de los precursores y los métodos de química fina, permiten modificar la morfología de los cristales, reducir las temperaturas de calcinación y dopar fácilmente al cerámico a fin de incrementar su densificación y propiedades piezoeléctricas [14, 17-19].

El objetivo de este trabajo es analizar diferentes condiciones de procesamiento del $\mathrm{K}_{1 / 2} \mathrm{Na}_{1 / 2} \mathrm{NbO}_{3}$ que permitan obtener un material con buenas propiedades y libre de fases secundarias. Se implementó el método de activación mecanoquímica de los precursores y posterior reacción en estado sólido. Se analizaron las principales condiciones de procesamiento (tiempo de molienda, temperaturas de calcinación y sinterizado), y se determinaron las propiedades dieléctricas y microestructurales de cada sistema.

\section{MATERIALES Y MÉTODOS}

El niobiato de sodio y potasio $\left(\mathrm{K}_{1 / 2} \mathrm{Na}_{1 / 2} \mathrm{NbO}_{3}\right)$ fue preparado mediante reacción en estado sólido, a partir de $\mathrm{K}_{2} \mathrm{CO}_{3}$ (Cicarelli 99,99\%; Argentina), $\mathrm{Na}_{2} \mathrm{CO}_{3}$ (Cicarelli 99,99\%; Argentina) y $\mathrm{Nb}_{2} \mathrm{O}_{5}$ (Aldrich 99,8\%; USA), siguiendo una relación estequiométrica $\mathrm{Na:K:Nb:O} \mathrm{de} \mathrm{1/2:1/2:1:3.}$

Los precursores de partida fueron molidos durante 2, $4 \mathrm{o}$ $6 \mathrm{~h}$ en un molino planetario (Fritsch, Pulverisette 7, 1000rpm) y medio alcohólico usando recipientes de circonia. A fin de asegurar la total descomposición de los carbonatos (20), el polvo resultante fue secado a $110^{\circ} \mathrm{C}$, tamizado y calcinado a 850 o $900^{\circ} \mathrm{C}$ durante 2 o $5 \mathrm{~h}$.

Se conformaron pastillas de $10 \mathrm{~mm}$ de diámetro y $1 \mathrm{~mm}$ de espesor por prensado uniaxial a $150 \mathrm{MPa}$, las que fueron sinterizadas a $1050,1075,1100,1125$ y $1150^{\circ} \mathrm{C}$ durante $2 \mathrm{~h}$, en atmósfera de aire, a una velocidad de calentamiento y enfriamiento de $3^{\circ} \mathrm{C} / \mathrm{min}$. Las pastillas sinterizadas fueron rectificadas con lijas de granulometría 320 y 600 a fin de eliminar desviaciones en la composición y mantener una superficie plana para el contacto eléctrico.

La densidad de las pastillas se determinó mediante el método de Arquímedes. La estructura cristalina y la microestructura de las muestras sinterizadas se caracterizaron mediante difracción de rayos X (DRX, Philips PW1830, $\mathrm{CuK}_{\alpha}$ ) y microscopía electrónica de barrido (SEM, JEOL 6460LV), respectivamente. Para el estudio mediante microscopía electrónica de barrido, las muestras fueron pulidas a espejo y tratadas térmicamente a $50^{\circ} \mathrm{C}$ por debajo de la temperatura de sinterizado, durante $20 \mathrm{~min}$. El espectro Raman fue obtenido usando un equipo Renishaw in Via Raman Microscope, con un láser de Argón de 514 nm y rendijas de difracción de 2400 líneas por milímetro.
El comportamiento dieléctrico fue estudiado sobre los discos, con electrodos de plata pintados sobre la superficie, a través de Espectroscopía de Impedancia (Hewlett Packard $4284 \mathrm{~A}$ ) entre $25 \mathrm{~Hz}$ y $1 \mathrm{MHz}$, y temperaturas de 30 a $410^{\circ} \mathrm{C}$. En todos los casos se empleó como porta muestras una celda Novocontrol BDS 1200.

Finalmente, los ciclos de histéresis (polarización vs campo eléctrico), fueron obtenidos a temperatura ambiente, en un baño de silicona, aplicando un campo eléctrico de $40 \mathrm{KV} / \mathrm{cm}$, de forma senoidal y una frecuencia de $50 \mathrm{~Hz}$.

\section{RESULTADOS Y DISCUSIÓN}

\subsection{Variables de Procesamiento}

\subsubsection{TEMPERATURA DE SINTERIZADO}

En una primera etapa y a fin de analizar el efecto de la temperatura de sinterizado sobre la microestructura y la densidad, se utilizaron muestras obtenidas luego de $6 \mathrm{~h}$ de mezclado de los precursores, en molino planetario, y posterior calcinación del polvo a $900^{\circ} \mathrm{C}$ durante 5 h. Las muestras fueron sinterizadas de 1050 a $1150^{\circ} \mathrm{C}$ a una velocidad de calentamiento y enfriamiento de $3^{\circ} \mathrm{C} / \mathrm{min}$.

En la Figura 1 se presentan los espectros de difracción de rayos $\mathrm{X}(\mathrm{A})$, junto con los valores de densidad y las micrografías de pastillas sinterizadas a 1050 y $1125^{\circ} \mathrm{C}$ (B). El espectro de DRX indica la presencia mayoritaria de la fase KNN (13-18), sólo las pastillas sinterizadas a $1150^{\circ} \mathrm{C}$ registran fases secundarias $\left(\mathrm{Nb}_{2} \mathrm{O}_{5^{\prime}}\right.$ $\mathrm{K}_{2} \mathrm{O}, \mathrm{K}_{3} \mathrm{NbO}_{4}$; JCPDS 15-0068, 23-0493, 30-964 respectivamente), probablemente debido a la evaporación del $\mathrm{K}_{2} \mathrm{O}$ (13). Cabe destacar que estas impurezas además de deteriorar las propiedades eléctricas, pueden provocar pequeños cambios en la estequiometría y llevar a la formación de fases indeseables que se disuelven fácilmente en presencia de humedad (13).

En la figura 1B se aprecia un incremento de la densidad con la temperatura de sinterizado, hasta alcanzar 4,32g/ $\mathrm{cm}^{3}$ para los sistemas sinterizados a $1150^{\circ} \mathrm{C}$. Dicha tendencia coincide con lo observado en las micrografías, dado que la muestra sinterizada a $1050^{\circ} \mathrm{C}$ presenta una microestructura porosa con pequeños granos de KNN que no llegaron a sinterizar correctamente, mientras que la muestra sinterizada a mayor temperatura presenta granos bien definidos de mayor tamaño y forma ortorrómbica. Por esta razón, se considera que la mejor temperatura de sinterizado es $1125^{\circ} \mathrm{C}$, dado que los materiales obtenidos a tal temperatura presentan altas densidades y se encuentran libres de fases secundarias.

\subsubsection{TIEMPO DE MOLIENDA Y CALCINACIÓN}

En esta sección se analiza el efecto del tiempo molienda (2 a $6 \mathrm{~h}$ ) y la temperatura y tiempo de calcinación $\left(850^{\circ} \mathrm{C}\right.$ por 5 h y $900^{\circ} \mathrm{C}$ por 2 y 5 h) sobre la densidad y estructura del KNN. Si bien en algunas investigaciones (20-21) se ha logrado reducir la temperatura de descomposición de los carbonatos a $700^{\circ} \mathrm{C}$ y obtener $\mathrm{KNN}$ libre de fases secundarias a menores temperaturas mediante la implementación de una etapa de molienda previa de los reactivos y una posterior molienda en molino de atrición durante 3 horas, en este trabajo se seleccionó una temperatura de 850 y $900^{\circ} \mathrm{C}$ para asegurar la completa descomposición de los componentes. 
En este trabajo, se empleó una etapa de molienda de $30 \mathrm{~min}$, posterior a la calcinación, en un molino planetario y se estableció una temperatura de sinterizado de $1125^{\circ} \mathrm{C}$ durante 2 horas. Esta segunda molienda facilitó el conformado de pastillas en los sistemas con menor tiempo de molienda inicial ( 2 o 4h), debido a que produce la ruptura de aglomerados (generados en la calcinación) y aumenta la reactividad al incrementar la superficie activa susceptibles a reaccionar en la etapa de sinterizado. La Figura 2 presenta los espectros de DRX y Raman de las muestras sinterizadas a $1125^{\circ} \mathrm{C}$. En ambas figuras se observa la presencia de la fase KNN libre de impurezas o fases secundarias $(13,15)$.

En el espectro Raman los picos de la región entre 100 y $160 \mathrm{~cm}^{-1}$ pueden ser asignados, debido a la similitud con los niobiatos alcalinos, a los modos de traslación de los cationes $\mathrm{K}^{+} / \mathrm{Na}^{+}$y la rotación del octaedro $\mathrm{NbO}_{6}^{-}(22-24)$. Los modos de vibración interna del octaedro aparecen en un amplio intervalo de frecuencias 170 a $900 \mathrm{~cm}^{-1}$ y dada la simetría del octaedro $(\mathrm{Oh})$ del grupo $\mathrm{NbO}_{6}^{-}$es de esperar que estén presentes los modos internos activos $\left(v_{1}\left(\mathrm{~A}_{1 \mathrm{~g}}\right), v_{2}\left(\mathrm{E}_{\mathrm{g}}\right)\right)$; mientras que probablemente los modos inactivos $\left(v_{3}\left(\mathrm{~F}_{1 \mathrm{u}}\right), v_{4}\left(\mathrm{~F}_{1 \mathrm{u}}\right), v_{5}\left(\mathrm{~F}_{2 \mathrm{~g}}\right), v_{6}\left(\mathrm{~F}_{2 \mathrm{u}}\right)\right)$ pueden
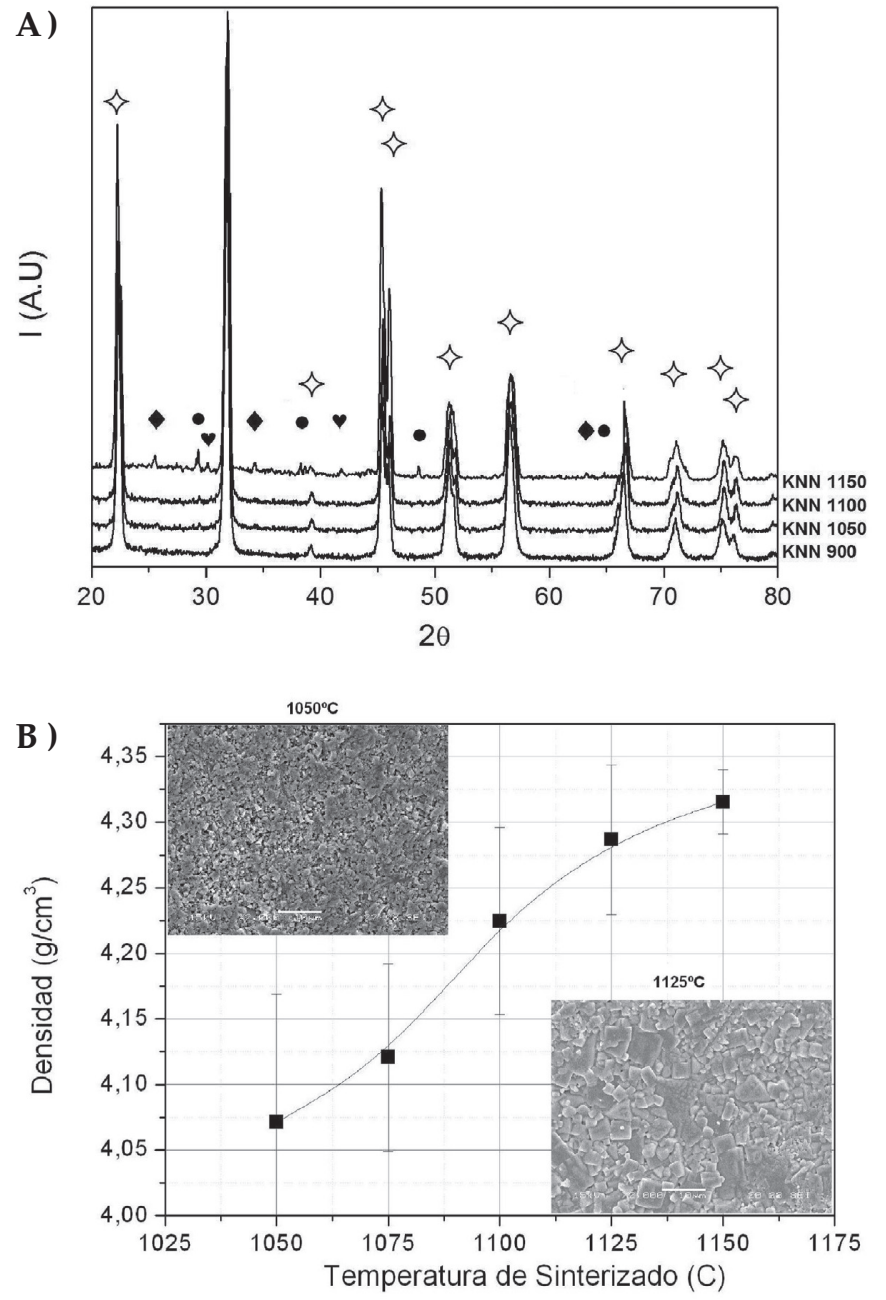

Figura 1. A) Patrón de difracción de rayos $\mathrm{X}(\mathrm{DRX})$ del polvo calcinado a $900^{\circ} \mathrm{C}$ y de las muestras sinterizadas a 1050,1100 y $1150^{\circ} \mathrm{C}$; B) valores de densidad y micrografías de pastillas sinterizadas a 1050 y $1125^{\circ} \mathrm{C}$. $\diamond \mathrm{KNN}$ [14-18]; $\mathrm{Nb}_{2} \mathrm{O}_{5}$ (JCPDS 37-1464); • $\mathrm{K}_{2} \mathrm{O}$ (JCPDS 23-0493); $\bullet \mathrm{K}_{3} \mathrm{NbO}_{4}$ (JCPDS 23-1345). ser detectados como dispersiones débiles (Figura 2B). No se observó desplazamiento ni ensanchamiento de los picos, que pudieran ser atribuidos a desordenes locales en la coordinación de los cationes dentro de la estructura o al incremento de la fuerza de enlace. No obstante, se aprecia una reducción de intensidad en los sistemas calcinados durante menor tiempo, probablemente debido a la presencia de fases menos cristalinas.

En la Figura 3 se aprecian las micrografías del polvo en función de las variables de procesamiento. En general, todos los sistemas presentan tamaños de partículas menores que $<1 \mu \mathrm{m}$, y en algunos casos se observó, a menores magnificaciones, la presencia de partículas de gran tamaño, junto a partículas pequeñas y con un alto grado de aglomeración. Este hecho se manifiesta particularmente en las muestras calcinadas a $900^{\circ} \mathrm{C}$ por $2 \mathrm{~h}$ y las molidas durante $4 \mathrm{~h}$ que además revelan una distribución menos homogénea.

\subsection{Microestructura y propiedades dieléctricas}

A fin de obtener información sobre la microestructura se realizaron micrografías de los sistemas conformados luego
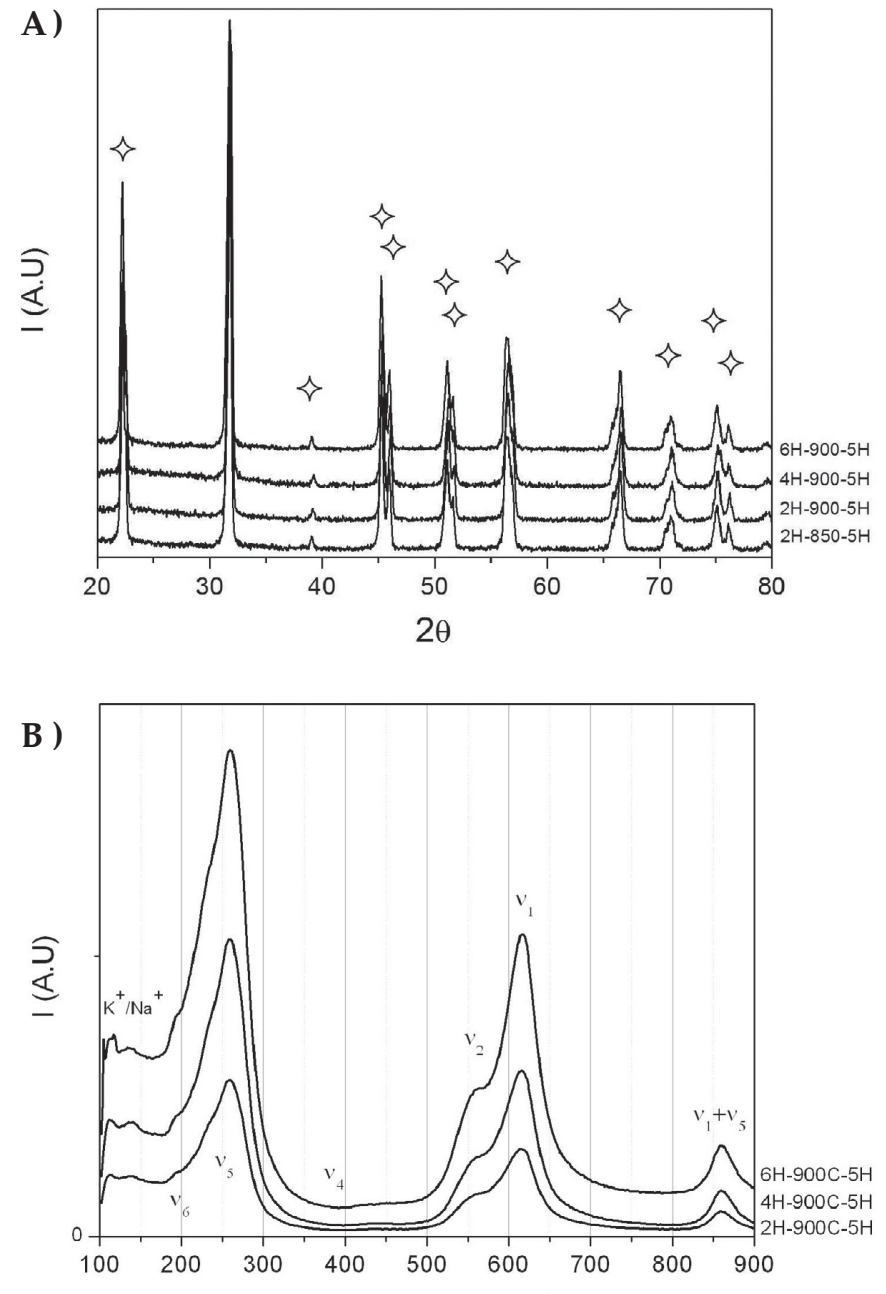

Figura 2. A) Patrón de difracción de rayos $\mathrm{X}$ de pastillas molidas por 2 a 6 h y calcinadas a 850 y $900^{\circ} \mathrm{C}$ durante 5 h ( $\diamond \mathrm{KNN}$ [14-15]); y B) Espectro Raman de pastillas molidas por 2 a $6 \mathrm{~h}$, calcinadas a $900^{\circ} \mathrm{C}$ durante 5 h y sinterizadas a $1125^{\circ} \mathrm{C}$. 
TABLA 1. VALORES DE PERMITIVIDAD REAL Y TANGENTE DE PÉRDIDA DE MUESTRAS OBTENIDAS LUEGO DE 2 A 6H DE MOLIENDA DE LOS PRECURSORES, CALCINADAS A $900^{\circ} \mathrm{C}$ DURANTE 2 A $5 \mathrm{H}$ Y SINTERIZADAS A $1125^{\circ} \mathrm{C}$ (A $30^{\circ} \mathrm{C}$ Y $2500 \mathrm{~Hz}$ ).

\begin{tabular}{|c|c|c|c|c|c|}
\hline $\begin{array}{c}\text { Nombre } \\
\text { Mol (h) }\end{array}$ & $\begin{array}{c}\text { Temp } \\
\text { Cal }\left({ }^{\circ} \mathbf{C}\right)\end{array}$ & $\begin{array}{c}\text { Tiempo } \\
\text { Cal (h) }\end{array}$ & $\varepsilon^{\prime}$ & tan $\delta$ \\
\hline KNN-2H-900C-2H & 2 & 900 & 2 & 820 & 0,369 \\
\hline KNN-2H-900C-5H & 2 & 900 & 5 & 1120 & 0,274 \\
\hline KNN-4H-900C-2H & 4 & 900 & 2 & 980 & 0,527 \\
\hline KNN-4H-900C-5H & 4 & 900 & 5 & 1035 & 0,096 \\
\hline KNN-6H-850C-5H & 6 & 850 & 5 & 760 & 0,057 \\
\hline KNN-6H-900C-2H & 6 & 900 & 2 & 1145 & 0,2673 \\
\hline KNN-6H-900C-5H & 6 & 900 & 5 & 2245 & 0,4732 \\
\hline
\end{tabular}

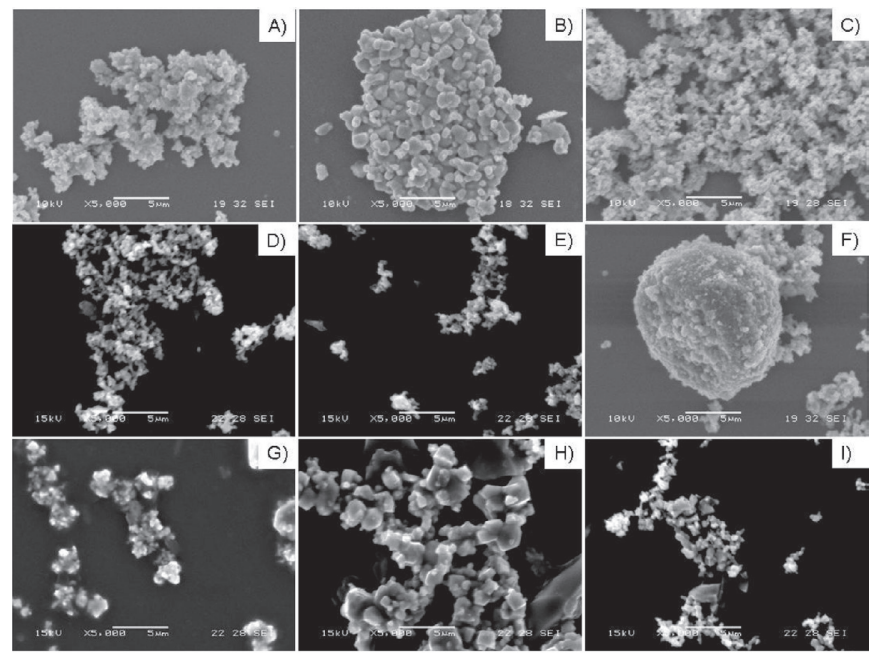

Figura 3. Imágenes de microscopía electrónica de barrido del polvo obtenido luego de 2 ( $\mathrm{A}, \mathrm{B}$ y C $), 4$ (D, E y F) y $6 \mathrm{~h}(\mathrm{G}, \mathrm{H}$ y I) de molienda de los precursores, calcinados a $850^{\circ} \mathrm{C}$ por $5 \mathrm{~h}\left(\mathrm{~A}, \mathrm{D}\right.$ y G) y $900^{\circ} \mathrm{C}$ por $2 \mathrm{~h}(\mathrm{~B}, \mathrm{E}$ y H) y $5 \mathrm{~h}(\mathrm{C}, \mathrm{F}$ y I).
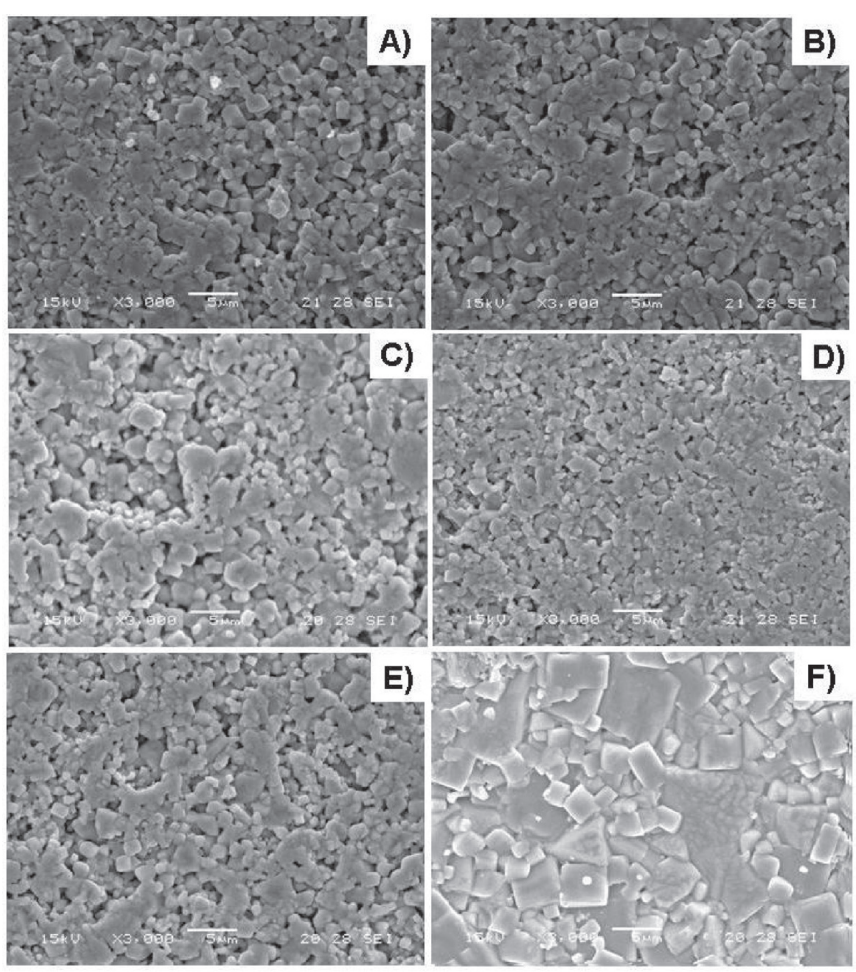

Figura 4. Imágenes de microscopía electrónica de barrido de pastillas obtenidas luego de $2 \mathrm{~h}$ (A y B), 4h (C y D) 6h (E y F) de molienda de los precursores, calcinados a $900^{\circ} \mathrm{C}$ por $2 \mathrm{~h}(\mathrm{~A}, \mathrm{C}$ y E) y $5 \mathrm{~h}(\mathrm{~B}, \mathrm{D}$ y F). de 2 a 6 h de molienda de los precursores, calcinados a $900^{\circ} \mathrm{C}$ por 2 y 5 h y sinterizados a $1125^{\circ} \mathrm{C}$ (Figura 4). Se observa que el tiempo de calcinación tuvo un efecto importante en la microestructura de las diferentes muestras. En general, todos los sistemas muestran granos pequeños $(\sim 1 \mu \mathrm{m})$ que resultan más finos y definidos a medida que aumenta el tiempo de molienda. No obstante, la muestra obtenidas luego $6 \mathrm{~h}$ de molienda y calcinada a $900^{\circ} \mathrm{C}$ durante 5 h, presenta una morfología con granos de mayor tamaño, forma cúbica y menor porosidad remanente que el resto de los sistemas. Lo que se puede atribuir al mayor crecimiento de grano por la calcinación a $900^{\circ} \mathrm{C}$ durante mayor tiempo.

La Tabla 1 muestra los valores permitividad real y tangente de pérdida de estos sistemas. Los materiales calcinados a $900^{\circ} \mathrm{C}$ durante tiempos cortos presentaron bajos valores permitividad, en contraste a los sistemas calcinados a $900^{\circ} \mathrm{C}$ durante 5 h que registraron valores más altos de permitividad. A partir de la observación microestructural se confirma que las muestras calcinadas a $900^{\circ} \mathrm{C}$ por $2 \mathrm{~h}$ revelaron estructuras más abiertas y con pequeños granos $(<1 \mu \mathrm{m})$ que no lograron densificar correctamente. Las muestras conformadas luego de $6 \mathrm{~h}$ de molienda y calcinadas a $900^{\circ} \mathrm{C}$ por $5 \mathrm{~h}$ presentaron
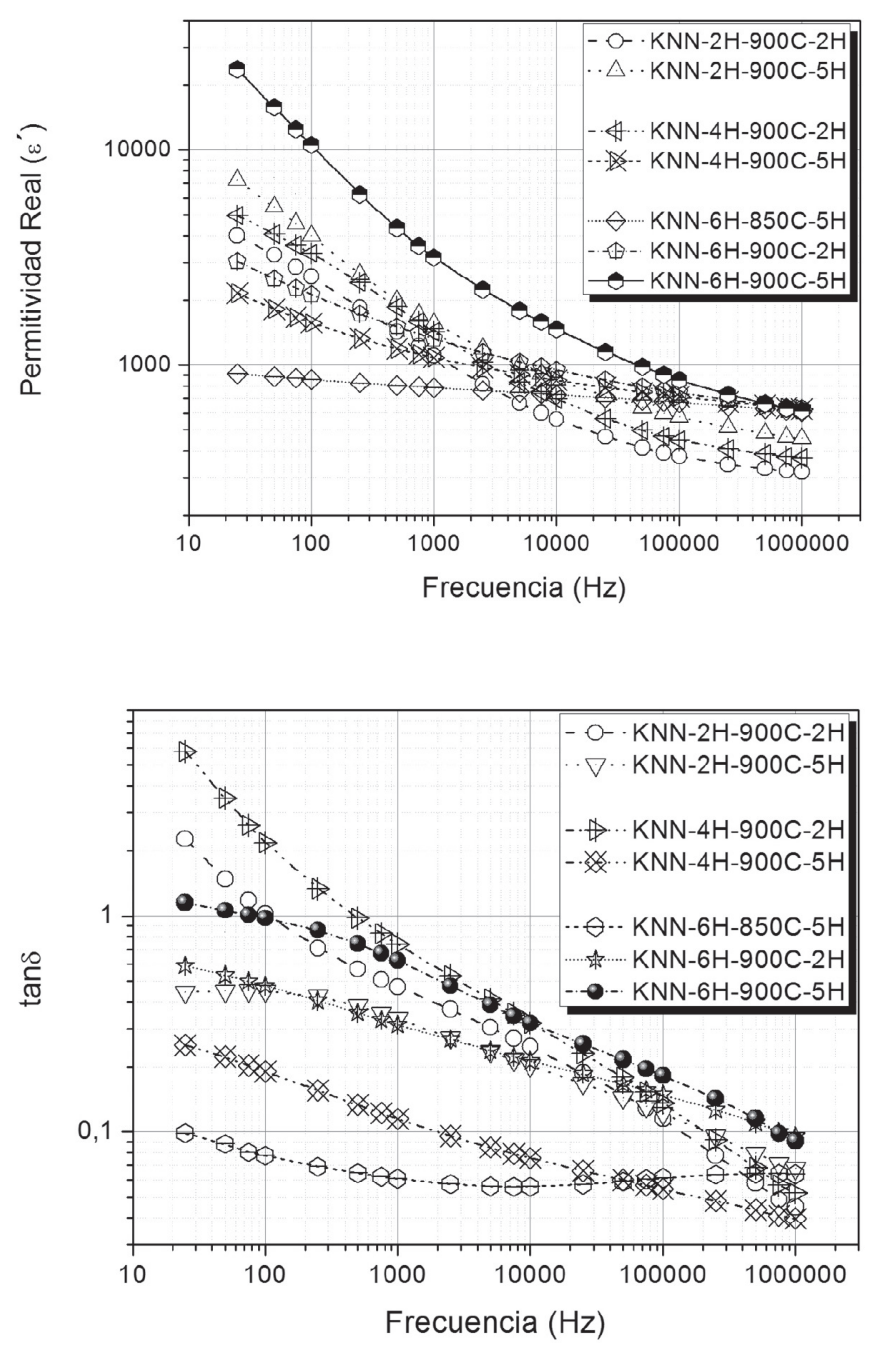

Figura 5. Permitividad real y tangente de pérdida a $30^{\circ} \mathrm{C}$ de muestras obtenidas luego de 2 a $6 \mathrm{~h}$ de molienda, calcinadas a 850 a $900^{\circ} \mathrm{C}$ durante 2 a 5 h y sinterizadas a $1125^{\circ} \mathrm{C}$. 
los valores de permitividad real y tangente de pérdida más altos, debido a una mejor distribución de tamaño de partículas y reactividad del polvo (Figura 3I). Cabe destacar que estos especímenes también mostraron los más altos valores densidad.

En la Figura 5 se aprecian los valores permitividad real y tangente de pérdida de cada sistemas $\left(a 30^{\circ} \mathrm{C}\right)$, incluyendo las muestras conformadas luego de $4 \mathrm{~h}$ de molienda. Se observa que los valores de permitividad y tangente de pérdida están altamente influenciados por la frecuencia, siendo estos últimos relativamente altos a bajas frecuencias. Probablemente debido a efectos de conductividad $d c$ favorecidos por un exceso de vacancias de oxígeno que no pudieron ser eliminadas durante el proceso de sinterizado. La muestra KNN-6H-900$5 \mathrm{H}$ registró los valores de permitividad real más elevados, mientras el sistema KNN-6H-850-5H las pérdidas más bajas.

La fase antiferroeléctrica $\mathrm{NaNbO}_{3}$ posee varias transiciones en el intervalo de -100 a $650^{\circ} \mathrm{C}$, sin embargo el $\mathrm{KNbO}_{3}$ combinado con $\mathrm{NaNbO}_{3}$ produce una nueva fase ferroeléctrica con transiciones a 200 y $420^{\circ} \mathrm{C}$, correspondientes a las transiciones de ortorrómbica a tetragonal y tetragonal (ferroeléctrica) a cúbica (paraeléctrica), respectivamente (25-
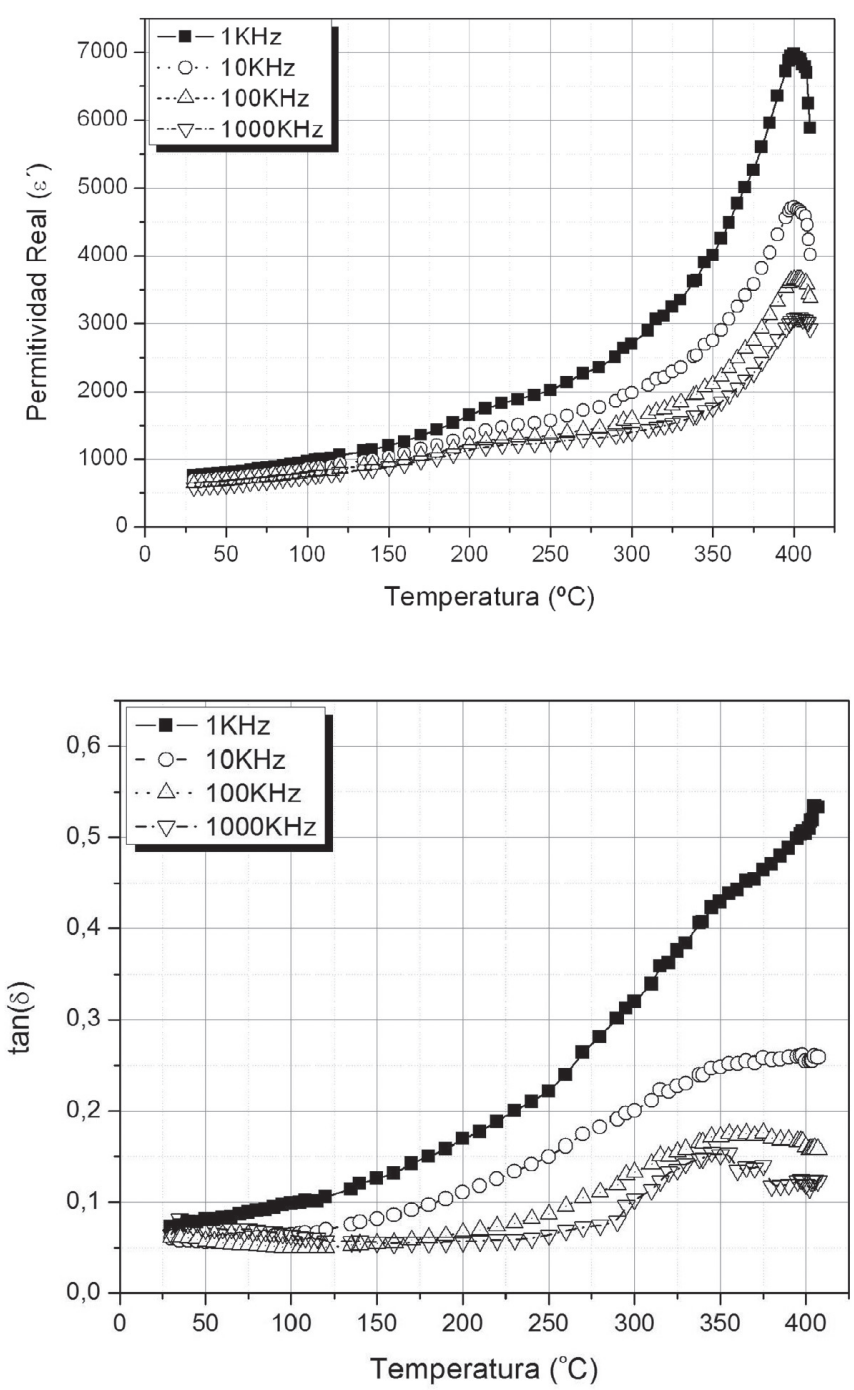

Figura 6. Permitividad real y tangente de pérdida en función de la temperatura para KNN-6H-900-5H (a 1, 10, 100 y 1000KHz).
27). En este sentido y si bien todas las muestras presentaron comportamientos similares con la temperatura, en la figura 6 se visualiza la variación de la permitividad real y la tangente de pérdida de la muestra $\mathrm{KNN}-6 \mathrm{H}-900-5 \mathrm{H}$, en función de la temperatura a diferentes frecuencias. Los valores de permitividad decrecen con el aumento de la frecuencia debido a procesos de relajación tipo Debye, observando una notable diferencia entre 1 y $10 \mathrm{KHz}$ (superiores a las diferencias entre 10, 100 y $1000 \mathrm{KHz}$ ). Los valores de permitividad aumentan con la temperatura hasta alcanzar un pico en la temperatura de Curie $\left(\mathrm{T}_{c}\right)$ a aproximadamente $400^{\circ} \mathrm{C}$, incremento igualmente registrado en la curva de tangente de pérdida (14, 26-28). A $210^{\circ} \mathrm{C}$ también se observa un suave pico en la curva de permitividad que está asociado a la transición de fase ortorrómbica a tetragonal (14).

Finalmente, en la figura 7 se observa el ciclo de histéresis del sistema KNN-6H-900-5H a temperatura ambiente, bajo un campo eléctrico de $40 \mathrm{KV} / \mathrm{cm}$ a $50 \mathrm{~Hz}$. En este sentido, las muestras registraron índices de polarización remante $(\mathrm{Pr})$ y polarización de saturación $\left(P_{S}\right)$ equivalentes a 7,9 y 13,45 $\mu \mathrm{C} / \mathrm{cm}^{2}$, respectivamente. Los que son valores similares a los registrados para sistemas tipo KNN $(24,28-29)$.

\section{CONCLUSIONES}

A partir de los resultados obtenidos se puede enunciar lo siguiente:

- La reacción en estado sólido previa activación y mezclado de los precursores permitió obtener la fase KNN pura. La temperatura facilitó el proceso de densificación, aunque las temperaturas máximas estuvieron limitadas por la descomposición de los constituyentes.

- La molienda post calcinación facilitó el procesamiento y permitió obtener pastillas luego de 2 y 4 h de activación mecanoquímica. Las muestras calcinadas durante $6 \mathrm{~h}$ a $900^{\circ} \mathrm{C}$, luego de la molienda, y sinterizadas a $1125^{\circ} \mathrm{C}$ durante 2 horas registraron alta densidad y permitividad y buenas características microestructurales. Estas observaciones pueden atribuirse a una buena distribución de partículas que optimizó el empaquetamiento del polvo.

- En general, los especímenes analizados fueron influenciados por la frecuencia, y registraron valores de pérdida dieléctrica relativamente altos.

- Los cerámicos obtenidos presentan la transición de fase ortorrómbica-tetragonal a $210^{\circ} \mathrm{C}$, mientras que la transición tetragonal-cúbica fue detectada a aproximadamente $400^{\circ} \mathrm{C}$.

- Finalmente, el análisis de la temperatura de sinterizado determinó que la temperatura óptima es de $1125{ }^{\circ} \mathrm{C}$, por presentar densidades superiores al $95 \%$ de la densidad teórica y no detectarse la presencia de fases secundarias. Para temperaturas $<1125{ }^{\circ} \mathrm{C}$ las cerámicas presentan densidades inferiores, y su microestructura está compuesta por tamaños de grano inferiores a $1 \mu \mathrm{m}$ y una alta concentración de porosidad abierta. Por otro lado, para las cerámicas sinterizadas a temperaturas $>1125^{\circ} \mathrm{C}$ se detectó la aparición de fases secundarias susceptibles de provocar cambios composicionales en el sistema. 


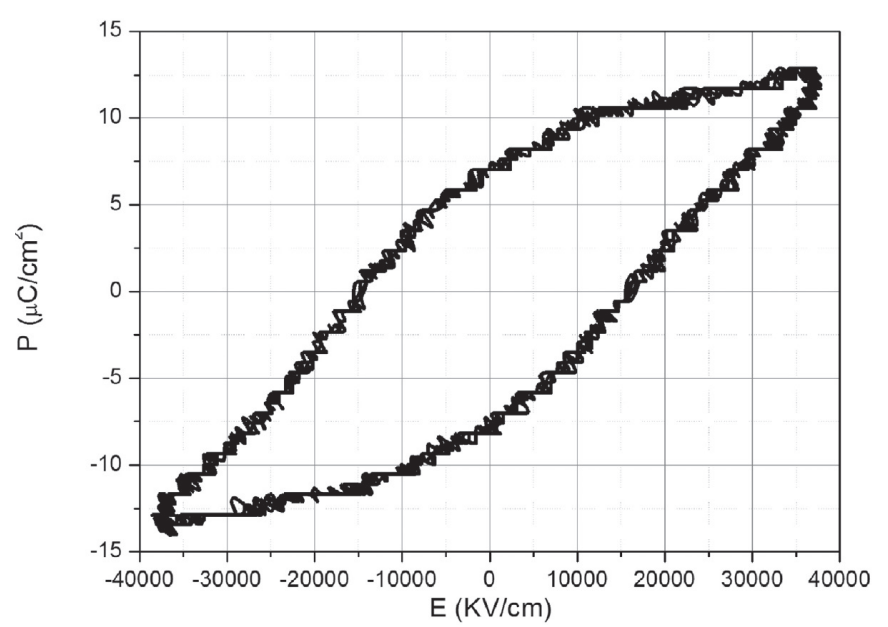

Figura 7. Ciclo de histéresis de KNN-6H-900-5H a temperatura ambiente. Condiciones de medida: campo-ac $40 \mathrm{KV} / \mathrm{cm}$, frecuencia $50 \mathrm{~Hz}$.

\section{AGRADECIMIENTOS}

Este trabajo ha sido posible gracias al apoyo económico e institucional del Consejo Nacional de Investigaciones Científicas y Técnicas (CONICET).

\section{REFERENCIAS}

1. B. Chu, D. Chen, G. Li, Q. Yin. «Electrical properties of $\mathrm{Na}_{1 / 2} \mathrm{Bi}_{1 / 2} \mathrm{TiO}_{3}-$ $\mathrm{BaTiO}_{3}$ ceramics». J. Eur. Ceram. Soc., 22 2115-2121 (2002).

2. Y. Li, W. Chen, Q. Xu., J. Zhou, Y. Wang, H. Sun. «Piezoelectric and dielectric properties of $\mathrm{CeO}_{2}$-doped $\mathrm{Bi}_{0.5} \mathrm{Na}_{0.44} \mathrm{~K}_{0.06} \mathrm{TiO}_{3}$ lead-free ceramics». Ceram. Int., 33 95-99 (2007).

3. A. Albareda, R. Perez, J. Garcia, D. Ochoa. «Comportamiento Intrínseco y Extrínseco de Piezoeléctricos». Bol. Soc. Esp. Ceram. V., 47 245-251 (2008)

4. Y. Guo, K. Kakimoto, H. Ohsato. «Dielectric and piezoelectric properties of lead-free $\left(\mathrm{Na}_{0.5} \mathrm{~K}_{0.5}\right) \mathrm{NbO}_{3}-\mathrm{SrTiO}_{3}$ ceramics». Solid State Commun., 129 279-284 (2004)

5. E Ringgaard, $\mathrm{T}$ Wurlitzer. «Lead-free piezoceramics based on alkali niobates». J. Eur. Ceram. Soc., 25 2701-2706 (2005).

6. Y-M Chiang, G. Farrey, A. Soukhojak. «Lead-free high-strain single-crystal piezoelectrics in the alkaline-bismuth-titanate perovskite family». Appl. Phys. Lett., 73 3683-6389 (1998).

7. S-E Park, S-J Chung. «Ferroic Phase Transitions in $\left(\mathrm{Na}_{1 / 2} \mathrm{Bi}_{1 / 2}\right) \mathrm{TiO}_{3}$ Crystals». J. Am. Ceram. Soc., 79 1290-1296 (1996).

8. Y. Shiratori, A. Magrz, C. Pithan. «Phase transformation of $\mathrm{KNaNb}_{2} \mathrm{O}$ induced by size effect». Chem. Phys. Lett., 391 288-292 (2004).

9. K. Kakimoto, I. Masuda, H. Ohsato. «Ferroelectric and Piezoelectric Properties of $\mathrm{KNbO}_{3}$ Ceramics Containing Small Amounts of $\mathrm{LaFeO}_{3}$ ". Jpn. J. Appl. Phys., 42 6102-6105 (2003).
10. D. Lin, D. Xiao, J. Zhu, P. Yu, H. Yan, L. Li. «Synthesis and piezoelectric properties of lead-free piezoelectric $\left[\mathrm{Bi}_{0.5}\left(\mathrm{Na}_{1-x-y} \mathrm{~K}_{x} \mathrm{Li}_{y}\right)_{0.5}\right] \mathrm{TiO}_{3}$ ceramics». Mater. Lett. 58 615-618 (2004).

11. Y. Saito, H. Takao, T. Tani, T. Nonoyama, K. Takatori, T. Homma, T. Nagoya, M. Nakamura. «Lead-free piezoceramics». Nature, 432 84-87 (2004).

12. E. Hollenstein, D. Damjanovic, M. Setter. «Temperature stability of the piezoelectric properties of Li-modified KNN ceramics». J. Eur. Ceram. Soc., 27 4093-4097 (2007)

13. F. Rubio-Marcos, P. Ochoa, J. Fernadez. «Sintering and properties of leadfree $(\mathrm{K}, \mathrm{Na}, \mathrm{Li})(\mathrm{Nb}, \mathrm{Ta}, \mathrm{Sb}) \mathrm{O}_{3}$ ceramics». J. Eur. Ceram. Soc., 27 4125-4129 (2007).

14. F. Rubio-Marcos, J.J. Romero, M.S. Martín-Gonzalez, J.F. Fernández. «Effect of stoichiometry and milling processes in the synthesis and the piezoelectric properties of modified KNN nanoparticles by solid state reaction». J. Eur. Ceram. Soc., 30 2763-2771 (2010)

15. Y. Gou, K. Kakimoto, H. Ohsato. «Phase transitional behavior and piezoelectric properties of $\left(\mathrm{Na}_{0.5} \mathrm{~K}_{0.5}\right) \mathrm{NbO}_{3}-\mathrm{LiNbO}_{3}$ ceramics». Appl. Phys. Lett., 85 4121-4123 (2004).

16. Shujun Zhanga, Ru Xiaa, R. Thomas Shrouta, Guozhong Zang, Jinfeng Wang. «Characterization of lead free $\left(\mathrm{K}_{0.5} \mathrm{Na}_{0.5}\right) \mathrm{NbO}_{3}-\mathrm{LiSbO}_{3}$ piezoceramic». Solid State Commun., 141 675-679 (2007).

17. H. Jacobsen, H-J Quenzer, B. Wagner, K. Ortner, T. Jung. «Thick PZT layers deposited by gas flow sputtering». Sensor Actuat., 135 23-27 (2007).

18. Y. Guo, K. Kakimoto, H. Ohsato. «Structure and Electrical Properties of Lead-Free $\left(\mathrm{Na}_{0.5} \mathrm{~K}_{0.5}\right) \mathrm{NbO}_{3}-\mathrm{BaTiO}_{3}$ Ceramics». Jpn. J. Appl. Phys., 43 66626666 (2004).

19. R. Wang, R Xie, T Sekita, Y. Shimoto, Y. Akimune, N. Hirosaki, M. Itoh. «Piezoelectric Properties of Spark-Plasma-Sintered $\left(\mathrm{Na}_{0.5} \mathrm{~K}_{0.5}\right) \mathrm{NbO}_{3}-$ $\mathrm{PbTiO}_{3}$ Ceramic». Jpn. J. Appl. Phys., 41 7119-7222 (2002).

20. F. Rubio-Marcos. «Estudio de Materiales Cerámicos Basados en el Sistema $(\mathrm{K}, \mathrm{Na}, \mathrm{Li})(\mathrm{Nb}, \mathrm{Ta}, \mathrm{Sb}) \mathrm{O}_{3}$ Relación entre la Estructura y las Propiedades Eléctricas». Tesis Doctoral, Universidad Autónoma de Madrid, España (2009).

21. F. Rubio-Marcos, J.J. Romero, J.F. Fernandez. «Effect of the temperature on the synthesis of $(\mathrm{K}, \mathrm{Na}) \mathrm{NbO}_{3}$-modified nanoparticles by a solid state reaction route». J. Nanopart. Res. 12, 2495-2502 (2010).

22. Y. Guo, H Luo, H Xu, X Zhou, X Pan, Z. Yin. «Ultra-high piezoelectric response in $<110>$-oriented polydomain $\mathrm{Pb}\left(\mathrm{Mg}_{1 / 3} \mathrm{Nb}_{2 / 3}\right) \mathrm{O}_{3}-\mathrm{PbTiO}_{3}$ single crystals». Appl. Phys. A Mater., 77 707-709 (2003).

23. Z. Shen, W. Wan, S. Tang, M. Kouk, «Raman scattering investigations of the antiferroelectric-ferroelectric phase transition of $\mathrm{NaNbO}_{3} »$. J. Raman Spectrosc., 29379 (1998).

24. R-C Chang, S-Y Chu, Y-F Lin, C-S Hong, P-C Kao, C-H Lu, «The effects of sintering temperature on the properties of $\left(\mathrm{Na}_{0.5} \mathrm{~K}_{0.5}\right) \mathrm{NbO}_{3}-\mathrm{CaTiO}_{3}$ based lead-free ceramics». Sensor Actuat A-Phys, 138 355-360 (2007).

25. H. Birol, D. Damjanovic, N. Setter, «Preparation and characterization of $\left(\mathrm{K}_{05} \mathrm{Na}_{05}\right) \mathrm{NbO}_{3}$ ceramics», J. Eur. Ceram. Soc., 26 861-866 (2006).

26. S-Y Chu, W Water, Y-D Juang, J-T Liaw, S-B Dai, «Properties of (Na, K) $\mathrm{NbO}_{3}$ and $(\mathrm{Li}, \mathrm{Na}, \mathrm{K}) \mathrm{NbO}_{3}$ ceramic mixed systems», Ferroelectrics, 287 23-33 (2003).

27. Y. Guo, K. Kakimoto, H. Ohsato, « $\left(\mathrm{Na}_{0.5} \mathrm{~K}_{0.5}\right) \mathrm{NbO}_{3}-\mathrm{LiTaO}_{3}$ lead-free piezoelectric ceramics», Mater. Lett., 59 241-244 (2005).

28. Y. Chang, Z. Yang, X. Chao, R. Zhang, X. Li, «Dielectric and piezoelectric properties of alkaline-earth titanate doped $\left(\mathrm{K}_{05} \mathrm{Na}_{05}\right) \mathrm{NbO}_{3}$ ceramics», Mater. Lett., 61 785-789 (2007).

29. J Wu, D Xiao, Y Wang, J Zhu,W Shi, W Wu, B Zhang, J Li, «Phase structure, microstructure and ferroelectric properties of $(1-x)\left[\left(\mathrm{K}_{050} \mathrm{Na}_{050}\right)_{004} \mathrm{Li}_{006}\right]$ $\left(\mathrm{Nb}_{0 .} \mathrm{Sb}_{0}\right) \mathrm{O}_{3} \mathrm{CaTiO}_{3}$ lead-free ceramics», I Alloy Compd, 476 782-786 (2009). 